\title{
A Case Report - Volatile Metabolomic Signature of Malignant Melanoma using Matching Skin as a Control
}

\author{
Tatjana Abaffy ${ }^{1 *}$, Mecker Möller ${ }^{2}$, Daniel D. Riemer ${ }^{3}$, Clara Milikowski $^{4}$ and R. Anthony DeFazio ${ }^{5}$
}

${ }^{1}$ Molecular and Cellular Pharmacology, University of Miami, Miami, FI, USA

${ }^{2}$ Dewitt Daughtry Department of Surgery, Division of Surgical Oncology, University of Miami, Miami, FI, USA

${ }^{3}$ Marine and Atmospheric Chemistry, Rosenstiel School of Marine and Atmospheric Science, University of Miami, Miami, FI, USA

${ }^{4}$ Department of Pathology, University of Miami, Miami, FI, USA

${ }^{5}$ Department of Neurology, University of Miami, Miami, FI, USA

\begin{abstract}
Melanoma is the most serious form of skin cancer. The quest for melanoma diagnostic biomarkers is paramount since early detection of melanoma and surgical excision represent the only effective treatment of this capricious disease. Our recent study tested the hypothesis that melanoma forms a unique volatile signature that is different than control, healthy tissue. Here, we are reporting a case study, the analysis of the volatile metabolic signature of a malignant melanoma using matched, non-neoplastic skin tissue from the same patient as a control. This is a significant improvement in the methodology, since it is well known that diet, skin type, genetic background, age, sex and environment all contribute to individual variation in the skin volatile signature. In the present study, we have identified 32 volatile compounds; 9 volatile compounds were increased in melanoma when compared to normal skin and 23 volatile compounds were detected only in melanoma and not in normal skin. Out of these 32 compounds, 10 have been reported previously by our group, thus confirming our results and adding additional confidence in our untargeted metabolomics approach for detection of melanoma biomarkers.
\end{abstract}

Keywords: Melanoma; Volatile compounds; GC/MS; Biomarkers; Metabolites

\section{Introduction}

The quest for melanoma biomarkers is paramount. The incidence of melanoma is increasing and mortality rates have not been significantly reduced. Early detection of melanoma is crucial as early surgical excision is the only life saving approach. However, distinguishing between benign pigmented lesions and early melanomas can be difficult [1] and there is a need for reliable markers that would improve and help in the diagnosis of this aggressive disease.

Cancer cells are known to have a highly unique metabolic phenotype, with pronounced anabolic metabolism associated with high glycolytic flux, increased lactate production and synthesis of macromolecules $[2,3]$. The study of specific metabolites-biomarkers to identify cancer fingerprints or signatures can aid in cancer detection, prognosis and in assessment of pharmacodynamic effects of therapy [4]. Metabolomics, the study of small molecule metabolites, is a link between genotype and phenotype. Experimental evidence shows that most oncogenes regulate downstream targets that are either directly or indirectly connected to the metabolic regulation [5]. Oncogenes can rewire metabolism, as shown in the case of the mutated succinate dehydrogenase gene (Sdh5) that disrupts the Krebs cycle [6]. Alternatively, metabolism and metabolic changes in the microenvironment drive oncogene mutation, e.g. KRAS mutations occurring in the low glucose tumor microenvironment [7]. These and other studies suggest that by monitoring changes in metabolite levels, we might be able to both detect cancer and better understand cancer pathological processes and progression. In this context, nuclear magnetic resonance (NMR), gas chromatographymass spectrometry (GC/MS) and liquid chromatography-mass spectrometry (LC/MS) are the analytical techniques most often applied in metabolite analysis [8].

Our recent study tested the hypothesis that melanoma forms a unique volatile signature that is different than control, healthy tissue [9].
This novel approach to detect and identify volatile metabolites released from melanoma tissue has the potential to discover novel biomarkers for detection of melanoma, as well as to increase our understanding of metabolic processes of this malignant cancer. In our previous study we identified compounds that are preferentially expressed in both fresh and frozen melanoma samples, when compared to unmatched fresh nevi and frozen skin samples, respectively. Our previous study provided a "proof of principle" that a differential volatile profile for melanoma does indeed exist [9].

Here, we further expand and enhance our efforts to identify a melanoma volatile signature by reporting the case analysis of the volatile metabolic signature of a malignant melanoma using matched, non-neoplastic skin tissue from the same patient as a control. We employed an untargeted approach, as this represents an efficient tool for early detection of disease $[10,11]$. We used Head Space Solid Phase Micro-Extraction (HS-SPME) [12] to collect volatile compounds from $2 \mathrm{~mm}$ punch biopsy skin samples, and GC/MS to separate and analyze these volatiles [9]. In these complex samples, there are still a significant number of compounds whose identity could not be reliably identified using GC/MS. We used a similarity threshold of $60 \%$ with the NIST 2.0 mass spectral database to assign the identity of the compounds. Thus, all compounds with a similarity $\leq 60 \%$ were excluded from analysis. The total number of identified and unidentified compounds

*Corresponding author: Tatjana Abaffy, RMSB, Molecular and Cellula Pharmacology, Miller School of Medicine, University of Miami, Miami, FI 33136, Tel: 305 243-1508; Fax: 305 243-4555; E-mail: tabaffy@med.miami.edu

Received April 18, 2011; Accepted June 30, 2011; Published July 12, 2011

Citation: Abaffy T, Möller M, Riemer DD, Milikowski C, DeFazio RA (2011) A Case Report - Volatile Metabolomic Signature of Malignant Melanoma using Matching Skin as a Control . J Cancer Sci Ther 3: 140-000. doi:10.4172/1948-5956.1000076

Copyright: (C) 2011 Abaffy T, et al. This is an open-access article distributed under the terms of the Creative Commons Attribution License, which permits unrestricted use, distribution, and reproduction in any medium, provided the original author and source are credited. 
in the melanoma sample was 166 and 693, respectively. In the matched, normal skin sample we identified 132 volatile compounds and 500 were unidentified.

In summary, we identified 32 compounds; 9 volatile compounds were increased in melanoma and 23 volatile compounds were detected only in melanoma and not in normal skin. Out of these 32 compounds, 10 have been reported previously [9], thus confirming our results and adding additional confidence in our untargeted metabolomics approach for detection of volatile compounds in melanoma.

\section{Material and Methods}

Biopsy samples were obtained in accordance with an approved University of Miami Institutional Review Board (IRB) protocol (No. 2006117). Fresh melanoma and skin samples were collected with a 2-mm punch device. Volatile collection and analysis was done as described in [9]. Briefly, we used HS-SPME (head space-solid phase microextraction) method to collect the volatiles. The headspace was sampled with a polydimethysiloxane-divinylbenzene fiber for one hour at room temperature (65- $\mu \mathrm{m}$ PDMS-DVB, Cat No. 57344-U, Supelco, Bellefonte, PA, USA). After volatile collection, samples were put into formalin and hematoxylin/eosin staining was done at at the Department of Pathology, University of Miami. The PDMS/DVB fiber with collected volatiles was directly injected onto a $0.75 \mathrm{~mm}$ i.d. injection port of Hewlett Packard 6890 gas chromatograph (Hewlett Packard, Avondale, PA) and chromatographed on a non-polar DB5MS column (model No. J\&W 128-5522, $25 \mathrm{~m} \mathrm{x} 0.2 \mathrm{~mm}$ i.d.x0.33 $\mu \mathrm{m}$ film) under the following temperature program: $40^{\circ} \mathrm{C}$ for two minutes followed by $6^{\circ} \mathrm{C} \mathrm{min}{ }^{-1}$ ramp to $270^{\circ} \mathrm{C}$ and hold for 5 minutes. Helium carrier gas flow was run in constant flow at $0.7 \mathrm{~mL} \mathrm{~min}{ }^{-1}$. As volatile compounds elute from the column, they were fragmented into ions (by electron ionization) and detected in the Agilent 5973, quadrupole mass spectrometer (in the full scan mode, 30-300 amu). We used the

\begin{tabular}{|l|l|}
\hline CAS/NIST & COMPOUNDS FOUND ONLY IN MELANOMA \\
\hline $2175-91-9$ & 1,3-Cyclopentadiene, 5-(1-methylethylidene)- \\
\hline $629-96-9$ & 1-Eicosanol \\
\hline $629-73-2$ & 1-Hexadecene \\
\hline $106-74-1$ & 2-Ethoxyethyl acrylate \\
\hline $28195-00-8$ & 2-Isopropylamino-4-methylbenzonitrile \\
\hline $14365-44-7$ & Adenosine, 5'-amino-5'-deoxy- \\
\hline $123-11-5$ & Benzaldehyde, 4-methoxy- \\
\hline $117-81-7$ & Bis(2-ethylhexyl) phthalate\#* \\
\hline $1678-91-7$ & Cyclohexane, ethyl- \\
\hline $112-31-2$ & Decanal \\
\hline $124-18-5$ & Decane* \\
\hline $6975-98-0$ & Decane, 2-methyl- \\
\hline $2847-72-5$ & Decane, 4-methyl-* \\
\hline $111-76-2$ & Ethanol, 2-butoxy- \\
\hline $75-21-8$ & Ethylene oxide* \\
\hline $75-12-7$ & Formamide \\
\hline $2213-23-2$ & Heptane, 2,4-dimethyl- \\
\hline $142-91-6$ & Isopropyl Palmitate\# \\
\hline $4282-42-2$ & Nonane, 1-iodo- \\
\hline $565-75-3$ & Pentane, 2,3,4-trimethyl- \\
\hline $314-84-7$ & Phthalic acid, isobutyl 4-octyl ester \\
\hline $6572-50-5$ & Spiro[bicyclo[2.2.1]hept-5-ene-2,1'-cyclopropane] \\
\hline $1120-21-4$ & Undecane*\# \\
\hline
\end{tabular}

Table 1: List of 23 volatile compounds found only in melanoma. In bold are volatiles previously identified as differentially expressed in respect to nevi $\left(^{*}\right)$ or normal skin (\#) [9].
AMDIS deconvolution algorithm freely available at www.amdis.net (Automated Mass Spectral Deconvolution and Identification software). All components from AMDIS analysis were searched in the NIST 2.0 database, with one reported hit per compound and with a minimum match factor set to $60 \%$.

\section{Results}

The patient, male, aged 49, was admitted to the hospital for excisional biopsy of melanoma lesion, one month after the diagnosis of melanoma in the primary care clinic was obtained. The initial diagnosis of Nodular Melanoma $\left(0.98 \mathrm{~mm}\right.$ Breslow thickness, $<1$ mitosis $\left./ \mathrm{mm}^{2}\right)$ was based on a biopsy from the \#1 distal lesion on the right forearm (Figure 1D). The patient was scheduled for additional excision of the \#2 proximal lesions (see Figure 1D). After informed consent was obtained for this study, a melanoma tissue sample was obtained from the \#2 proximal lesion. After volatile collection and analysis, this sample was sent for histologic analysis. It was positive for malignant melanoma (H\&E staining, Figure 1A, $0.78 \mathrm{~mm}$ Breslow thickness, <1 mitosis/ $\mathrm{mm}^{2}$, stage T1a, N0M0). A second punch biopsy of nearby healthy skin was performed at the site indicated in Figure 1D. Chromatograms obtained from these two sites are presented in Figure $1 \mathrm{~B}$ and F. The peaks in the chromatograms indicated the relative abundance of compounds eluting at the time indicated in the $\mathrm{x}$-axis. Compounds unique to the melanoma samples, with respect to the healthy skin control, are shown in Table 1. Compounds enriched in melanoma, with respect to the healthy skin control, are shown in Table 2.

Our analysis shows a marked 35 fold increase in 1-Hexadecanol in melanoma, as compared to the matching skin sample (Table 2), suggesting 1-Hexadecanol as a potential biomarker of melanoma. 1- Hexadecanol has been previously detected in skin emanations [13] and a high temperature GC/MS study of skin surface predominant lipids and fatty acids identified hexadecenoic acid (C16:1) and hexadecanoic acid (C16:0) [14]. The greatly increased level of 1-hexadecanol in the melanoma sample may reflect increased de novo fatty acid (FA) synthesis, a crucial metabolic alteration of cancer cells required for synthesis of new plasma membranes. Increased activity of fatty acid synthase (FAS, an enzyme responsible for the synthesis of fatty acids), has emerged as a phenotype common to most tumors associated with poor outcome [15]. FAS over-expression in melanoma has been correlated with Breslow thickness and overall poor survival $[16,17]$. In addition, lipids impact cancer cell growth and invasion. For example, lysophosphatidic acid acts through its receptors to stimulate cancer cell proliferation and survival $[2,18]$. Oncogene induced FA synthesis facilitates the formation of palmitate and palmitoleate. In our melanoma case, chemical reduction of abundant hexadecanoic acid results in increased levels of 1-hexadecanol and indicate the importance of palmitic acid (C16:0) and free fatty acid alcohol (1-hexadecanol). We also detected 1-hexadecene and isopropyl palmitate, derivatives of palmitic acid. Taken together, these results implicate increased fatty acid synthesis and metabolism in the progression of melanoma. Further studies on larger cohort are necessary to confirm this observation.

Methyled benzene (Benzene, 1,3,5-trimethyl) is also increased in the melanoma sample compared to normal skin sample (Table 2). This volatile has been previously found in skin [13] and we have previously detected the increased frequency of methylated benzenes in melanoma vs nevi [9].

The amide formamide was detected only in the melanoma sample (Table 1). Very few amides are found to be released from human skin [13] and to date, there are no reports of formamide detected 
Citation: Abaffy T, Möller M, Riemer DD, Milikowski C, DeFazio RA (2011) A Case Report - Volatile Metabolomic Signature of Malignant Melanoma using Matching Skin as a Control . J Cancer Sci Ther 3: 140-000. doi:10.4172/1948-5956.1000076

\begin{tabular}{|c|c|c|c|c|}
\hline CAS/NIST & COMPOUND & $\%$ TIC in M & \% TIC in $\mathrm{S}$ & RATIO M/S \\
\hline $117-84-0$ & 1,2-Benzenedicarboxylic acid, diisooctyl ester & 1.03 & 0.61 & 1.68 \\
\hline $36653-82-4$ & 1-Hexadecanol* & 3.29 & 0.09 & 35.90 \\
\hline 83834-59-7 & 2-Ethylhexyl trans-4-methoxycinnamate & 1.02 & 0.78 & 1.31 \\
\hline $108-67-8$ & Benzene, 1,3,5-trimethyl* & 0.12 & 0.03 & 3.41 \\
\hline $75-43-4$ & Dichlorodifluoromethane & 0.03 & 0.01 & 1.80 \\
\hline $112-40-3$ & Dodecane* & 0.13 & 0.11 & 1.27 \\
\hline $77422-34-5$ & N-Morpholinomethyl-isopropyl-sulfide & 0.32 & 0.23 & 1.37 \\
\hline $124-19-6$ & Nonanal\# & 0.30 & 0.11 & 2.79 \\
\hline $17301-32-5$ & Undecane, 4,7-dimethyl & 0.17 & 0.11 & 1.54 \\
\hline
\end{tabular}

Table 2. Compounds that showed $\geq 100 \%$ enrichment in melanoma compared to the healthy matched skin sample are listed together with the $\%$ Total lon Count, (\%TIC) and their ratio in Melanoma (M) vs Skin (S) - M/S. In order to compensate for differences, the signal intensity of each volatile compound was normalized to the TIC of all volatiles detected in the particular sample. Compounds in bold have been previously identified as significantly different in melanoma when compared to nevi $\left(^{*}\right)$ or to skin (\#) control [9].
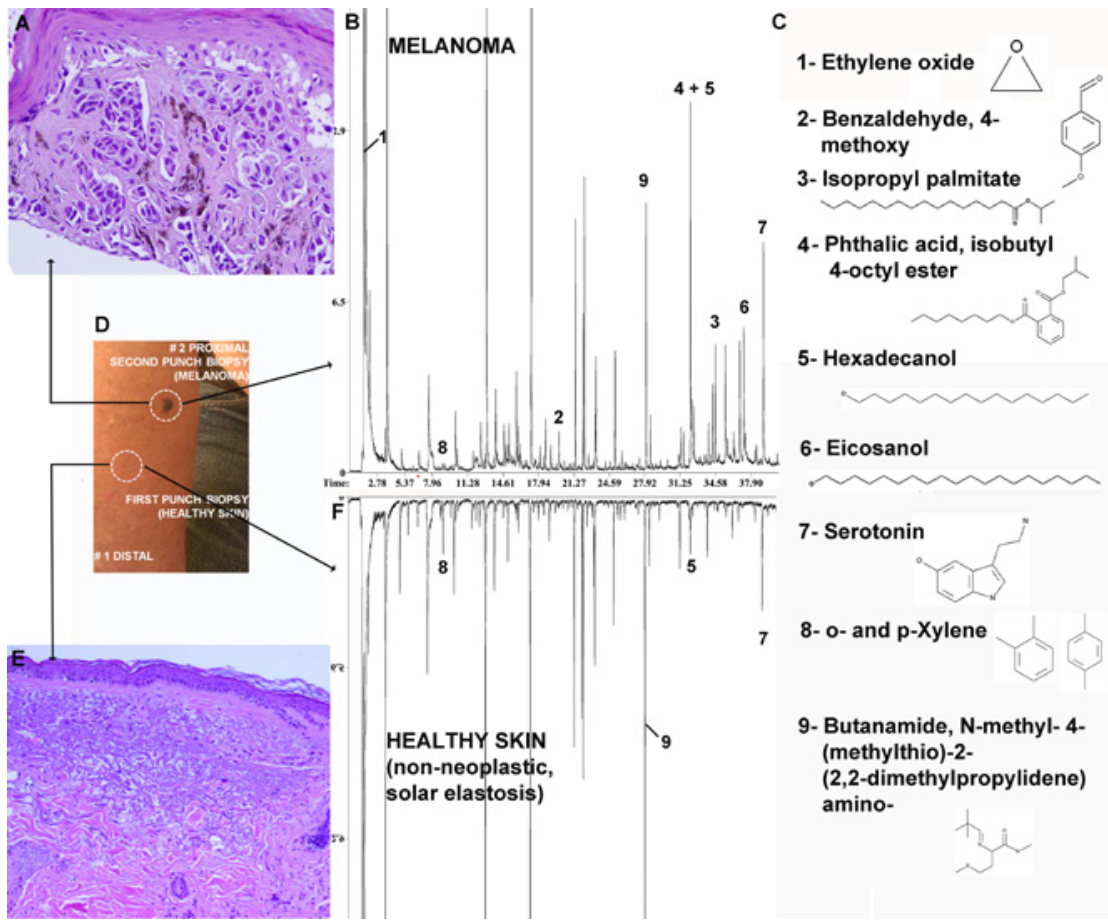

Figure 1: Comparison of volatile signatures from a malignant melanoma biopsy and nearby healthy non-neoplastic matching skin biopsy. A. Histology - H\&E staining of the \#2 proximal punch biopsy melanoma lesion (40X magnification). B. Full chromatogram of melanoma sample. Some compounds found to be differentially expressed in melanoma vs skin are numbered and indicated in the chromatograms. Their names and structures are presented in C. D. Biopsy sites from the right forearm.E. Histology - H\&E staining of healthy, non-neoplastic skin showing signs of solar elastosis. F. Full chromatogram of the non-neoplastic healthy matched skin sample.

from human skin. However, methane, ethylene and ethane were detected on human skin [19]. Biological oxidation of methane to $\mathrm{CO}_{2}$ proceeds sequentially through methanol, formaldehyde and formic acid. Previously, we detected a significant increase in formic acid in melanoma relative to the nevi group [9]. The Human Metabolome Database (http://www.hmdb.ca/metabolites/HMDB00142) lists formic acid as an intermediate in the metabolism of one-carbon compounds. It is a product of fatty acid oxidation [20] and it can also be produced by bacterial fermentation via heterofermenters, microorganisms that ferment glucose to multiple end products, such as acetic acid, ethanol, formic acid and $\mathrm{CO}_{2}$ [21]. Formic acid has also been detected in cerebrospinal fluid [22] and urine [23]. Since ammonia is known to be one component of human odor and sweat [24], it is easy to imagine the generation of formamide from the reaction of ammonia with formic acid. This may represent the underlying biochemical pathway for the production of formamide in melanoma. In addition, the ammonia necessary for formamide synthesis may have come from increased glutaminolysis known to be a characteristic of cancer cells [25]. Large quantities of glutamine are taken up by cancer cells, resulting in secretion of alanine and ammonium into the extracellular matrix [26,27]. Increased glutaminase activity (the enzyme that catabolizes glutamine for ATP through the TCA cycle) is documented in melanoma $[28,29]$. The biochemical origin of ammonium in our melanoma sample may be increased catabolism of glutamine which results in ammonia release. However, this is a hypothesis and needs to be fully investigated.

We detected ethylene oxide in melanoma (Table 1 and Figure $1 \mathrm{~B}$ and 1C). The metabolic precursor for ethylene oxide is ethylene, a natural body constituent. Ethylene was previously detected on human skin [19]. However, there are no data on endogenous ethylene formation in humans or animals. A human enzyme potentially responsible for 
ethylene synthesis is 1-aminocyclopropane-1-carboxylate synthase (ACC synthase) (E.C. 4.4.1.14); however it is reportedly inactive. The mutations of two amino-acid residues within the binding site of the enzyme are responsible for the lack of activity in the human ACC synthase [30]. Thus, it seems unlikely that the ethylene oxide in melanoma samples is a primary metabolite. Could it be produced via degradation of the primary metabolites and/or proteins and lipids within a cell? Intestinal bacteria are thought to give rise to ethylene, the precursor of ethylene oxide, via lipid peroxidation and metabolism $[31,32]$. Whether a similar mechanism exists in human skin remains to be explored. Ethylene oxide carcinogenicity studies have revealed that it is a direct-acting alkylating agent forming adducts and causing hydroxyethylation of DNA and proteins [33]. These adducts were initially used to monitor worker exposure to ethylene oxide, but it was soon realized that there are background levels of hemoglobin and DNA adducts in humans and experimental animals that have not been exposed to ethylene oxide. Additional studies showed that ethylene indeed can be formed in animals when they are fed with unsaturated lipids. Thus, possible endogenous sources of ethylene oxide and ethylene are lipid peroxidation, methionine oxidation and metabolism of intestinal bacteria [33]. Ethylene oxide is frequently used in sterilization of medical instruments (especially for sterile disposable medical devices), thus raising the question of external contamination from the punch biopsy device. We can rule out external contamination because ethylene oxide was not detectable in the skin sample acquired in parallel with the melanoma sample (data not shown).

What is the origin of Bis(2-ethylhexyl) phthalate and Phthalic acid, isobutyl 4-octyl ester (Table 1 and Figure 1C)? - Is it a contaminant? In general, phthalate esters have been widely used as plasticizers. Also, it has been shown that anticancer drugs, anthracyclines, metabolize to phthalic acids by oxidative degradation [34]. Our melanoma patient was not given any anthracycline therapy. Finally, Bis(2-ethylhexyl) phthalate was previously identified at an increased level in melanoma biopsies-compared to both normal skin and nevi, excluding the possibility of contamination [9].

We identified 1-eicosanol (Table 1 and Figure 1C), an alcohol containing 20 carbons (eicosa) as a volatile compound specific for melanoma. Eicosanoids are bioactive lipid metabolites derived from the metabolism of polyunsaturated fatty acids by cyclooxygenases, lipoxygenases, cytochrome $\mathrm{P} 450$ and nonenzymatic pathways [35]. They are synthesized by activated inflammatory cells. 12-(S) Hydroxy-5, 8, 11, 13-eicosatetraenoic acid (12-(S)-HETE ) and 15-(S)HETE) are monohydroxylated lipoxygenase derivatives of arachidonic acid. When $\left[1-{ }^{14} \mathrm{C}\right] 15-(\mathrm{S})$-HETE was incubated with Caco-2 cells (enterocytes derived from a human adenocarcinoma), about $10 \%$ was directly esterified into cellular lipids but most was beta-oxidized to ketone bodies, $\mathrm{CO}_{2}$, and acetate (55\%). The resulting acetate was then incorporated into the synthesis of new fatty acids, primarily $\left[{ }^{14} \mathrm{C}\right]$ palmitate. Thus, it is possible that the derivative of palmitate, isopropyl palmitate (Figure 1C and Table 1), in our melanoma case also originated from the increased metabolism of arachidonic acid in cancer cells (in addition to the oncogene induced de novo FA synthesis from glucose proposed earlier).

We detected increased nonanal in melanoma (Table 2). Previously, we identified nonanal as a potential melanoma biomarker [9]. Further studies on a larger set of melanoma samples will be necessary to clearly establish the role of this aldehyde in the malignant process. In this study, 1-iodo nonane was detected only in melanoma (Table 1). Previously, we detected nonane at a significantly increased frequency in melanoma when compared to the nevi [9].
Our analysis also identified undecane only in the melanoma sample (Table 1), while methylated undecane was increased in melanoma compared to the skin sample (Table 2).

\section{Discussion}

In the present case control study we used healthy skin from the same patient as a control tissue. Thus, we have reduced the potential for confounding variation or "the noise" due to age, nutritional status, metabolic, genetic and environmental influences that may arise between test and control. This study design also limits the impact of environmental or experimental contamination because the samples were collected in parallel from the same patient using the same equipment. However, further studies enrolling a large number of patients and using matched skin controls are necessary to elaborate and validate the full spectrum of melanoma biomarkers. In conclusion, out of 32 volatile compounds listed in Table 1 and Table 2, we detected 10 compounds (in bold) that have also been previously shown to be significantly different from control fresh nevi $\left(^{*}\right)$ and control frozen normal skin (\#) [9]. These volatile compounds are candidate volatile biomarkers of melanoma. As mentioned previously, many volatiles released from both melanoma and normal skin sample remain unidentified. The high resolution separation and exact mass determination from the complex matrixes such as skin will require a more sensitive mass spectrometer (e.g. TOF MS) to accurately identify these many additional compounds.

The exact biochemical origin of the detected volatiles is unknown. It is highly likely that these volatiles represent either primary or secondary metabolites. As primary metabolites, they may be involved in the communication between cells, in the transfer of the information from damaged or metabolically inactive cells. They may also signal a stress response and/or they may directly signal a cancer phenotype. Some volatiles may also represent signals involved in the recruitment of the immune response. As secondary metabolites the volatiles may result from transformation, oxidation or breakdown of primary metabolites or from the degradation of proteins and plasma membranes resulting from necrosis. Metabolites can originate from any cell type within skin, such as melanocytes, keratinocytes, fibroblasts, lymphocytes, macrophages, dendritic and/or myeloid cells. It is known that during early stages of melanoma progression, innate and adaptive immune cells are recruited to the tumor site (e.g. macrophages). Eosinophil recruitment/infiltration in tumors has been documented using B16-F10 melanoma cells [36]. Although the role of eosinophils is unclear, their accumulation in the necrotic region suggests that they may promote necrosis [37]. In addition, structural components of extracellular matrix (ECM) including collagens, elastins and soluble factors like cytokines, chemokines and polypeptide growth factors released either from neoplastic or non-neoplastic cells and their degradation products may also contribute to the melanoma volatile metabolic signature. It is known that tumor associated-macrophages release matrix metalloproteinase (MMP) and elastase and are thus actively involved in the breaking and remodeling of the ECM [38] Collagen degradation is necessary for melanoma tumor invasion and progression. Migrating cells must cross the dermis, which is rich in type I collagen; thus both serine proteinases and MMP are required for tissue invasion by cancer cells $[39,40]$. There are many different biochemical pathways that might play a significant role in shaping a volatile signature of melanoma, and although it is irrelevant from the diagnostic perspective, studying underlying biochemistry of metabolic changes in melanoma, and cancer in general, represents a fascinating new research direction. 
Citation: Abaffy T, Möller M, Riemer DD, Milikowski C, DeFazio RA (2011) A Case Report - Volatile Metabolomic Signature of Malignant Melanoma using Matching Skin as a Control . J Cancer Sci Ther 3: 140-000. doi:10.4172/1948-5956.1000076

\section{Competing Interests}

The authors have declared that no competing interests exist.

\section{Authors' Contributions}

Conceived and designed the experiment: T.A. Performed the experiment: T.A. Analyzed the data: T.A. and R.A.D. Contributed materials/analysis tools: M.M. D.D. R. and C.M. T.A. and R.A.D. wrote the paper.

\section{Acknowledgments and Funding}

This work was supported by grants from the University of Miami (T.A.), Florida Biomedical Bankhead-Coley Research Program (T.A.) and from National Institutes of Health (NIH National Cancer Institute (NCI) R21 CA132046 (T.A.).

\section{References}

1. Corona R, Mele A, Amini M, De Rosa G, Coppola G, et al. (1996) Interobserver variability on the histopathologic diagnosis of cutaneous melanoma and other pigmented skin lesions. J Clin Oncol 14: 1218-1223.

2. Fritz $V$, Fajas $L$ (2010) Metabolism and proliferation share common regulatory pathways in cancer cells. Oncogene 29: 4369-4377.

3. Jezek P, Plecita-Hlavata L, Smolkova K, Rossignol R (2010) Distinctions and similarities of cell bioenergetics and the role of mitochondria in hypoxia, cancer, and embryonic development. Int J Biochem Cell Biol 42: 604-622.

4. Spratlin JL, Serkova NJ, Eckhardt SG (2009) Clinical applications of metabolomics in oncology: a review. Clin Cancer Res15: 431-440.

5. Locasale JW, Cantley LC (2009) Vander Heiden MG: Cancer's insatiable appetite. Nat Biotechnol 27: 916-917.

6. Hao HX, Khalimonchuk O, Schraders M, Dephoure N, Bayley JP, et al. (2009) $\mathrm{SDH} 5$, a gene required for flavination of succinate dehydrogenase, is mutated in paraganglioma. Science 325: 1139-1142.

7. Yun J, Rago C, Cheong I, Pagliarini R, Angenendt P, et al. (2009) Glucose deprivation contributes to the development of KRAS pathway mutations in tumor cells. Science 325: 1555-1559.

8. Fernie AR, Trethewey RN, Krotzky AJ, Willmitzer L (2004) Metabolite profiling: from diagnostics to systems biology. Nat Rev Mol Cell Biol 5: 763-769.

9. Abaffy T, Duncan R, Riemer DD, Tietje O, Elgart G, et al. (2010) Differential volatile signatures from skin, naevi and melanoma: a novel approach to detect a pathological process. PLoS One 5: e13813.

10. Ackermann BL, Hale JE, Duffin KL (2006) The role of mass spectrometry in biomarker discovery and measurement. Curr Drug Metab 7: 525-539.

11. Sreekumar A, Poisson LM, Rajendiran TM, Khan AP, Cao Q, et al. (2009) Metabolomic profiles delineate potential role for sarcosine in prostate cancer progression. Nature 457: 910-914

12. Pawliszyn J (2000) Theory of solid-phase microextraction. J Chromatogr Sc 38: $270-278$

13. Bernier UR, Kline DL, Barnard DR, Schreck CE, Yost RA (2000) Analysis of human skin emanations by gas chromatography/mass spectrometry. 2. Identification of volatile compounds that are candidate attractants for the yellow fever mosquito (Aedes aegypti). Anal Chem 72: 747-756.

14. Michael-Jubeli R, Bleton J, Baillet-Guffroy A (2011) High-temperature gas chromatography-mass spectrometry for skin surface lipids profiling. J Lipid Res 52: 143-151.

15. Kuhajda FP (2006) Fatty acid synthase and cancer: new application of an old pathway. Cancer Res 66: 5977-5980.

16. Innocenzi D, Alo PL, Balzani A, Sebastiani V, Silipo V, et al. (2003) Fatty acid synthase expression in melanoma. J Cutan Pathol 30: 23-28.

17. Kapur P, Rakheja D, Roy LC, Hoang MP (2005) Fatty acid synthase expression in cutaneous melanocytic neoplasms. Mod Pathol 18: 1107-1112.

18. Bauer DE, Hatzivassiliou G, Zhao F, Andreadis C, Thompson CB (2005) ATP citrate lyase is an important component of cell growth and transformation. Oncogene 24: 6314-6322.

19. Nose K, Nunome Y, Kondo T, Araki S, Tsuda T (2005) Identification of gas emanated from human skin: methane, ethylene, and ethane. Anal Sci 21: 625628.
20. Poulos A, Sharp P, Singh H, Johnson DW, Carey WF, et al. (1993) Formic acid is a product of the alpha-oxidation of fatty acids by human skin fibroblasts: deficiency of formic acid production in peroxisome-deficient fibroblasts. Biochem J 292: 457-461.

21. Baron S (1996) Medical microbiology. University of Texas Medical Branch at Galveston.

22. Wishart DS, Lewis MJ, Morrissey JA, Flegel MD, Jeroncic K, et al. (2008) The human cerebrospinal fluid metabolome. J Chromatogr B Analyt Technol Biomed Life Sci 871: 164-173.

23. Bales JR, Higham DP, Howe I, Nicholson JK, Sadler PJ (1984) Use of highresolution proton nuclear magnetic resonance spectroscopy for rapid multicomponent analysis of urine. Clin Chem 30: 426-432.

24. Smallegange RC, Qiu YT, van Loon JJ, Takken W (2005) Synergism between ammonia, lactic acid and carboxylic acids as kairomones in the host-seeking behaviour of the malaria mosquito Anopheles gambiae sensu stricto (Diptera: Culicidae). Chem Senses 30: 145-152.

25. Feron O (2009) Pyruvate into lactate and back: from the Warburg effect to symbiotic energy fuel exchange in cancer cells. Radiother Oncol 92: 329-333.

26. Levine AJ, Puzio-Kuter AM (2010) The control of the metabolic switch in cancers by oncogenes and tumor suppressor genes. Science 330: 1340-1344.

27. Eng CH, Yu K, Lucas J, White E, Abraham RT (2010) Ammonia derived from glutaminolysis is a diffusible regulator of autophagy. Sci Signal 3: ra31.

28. DeBerardinis RJ, Mancuso A, Daikhin E, Nissim I, Yudkoff M, et al. (2007) Beyond aerobic glycolysis: transformed cells can engage in glutamine metabolism that exceeds the requirement for protein and nucleotide synthesis. Proc Natl Acad Sci U S A 104: 19345-19350.

29. Zacharias DP, Lima MM, Souza AL Jr., de Abranches Oliveira Santos ID Enokiara M, et al. (2003) Human cutaneous melanoma expresses a significant phosphate-dependent glutaminase activity: a comparison with the surrounding skin of the same patient. Cell Biochem Funct 21: 81-84.

30. Koch KA, Capitani G, Gruetter MG, Kirsch JF (2001) The human cDNA for a homologue of the plant enzyme 1-aminocyclopropane-1-carboxylate synthase encodes a protein lacking that activity. Gene $272: 75-84$.

31. Tornqvist M, Gustafsson B, Kautiainen A, Harms-Ringdahl M, Granath F, et al. (1989) Unsaturated lipids and intestinal bacteria as sources of endogenous production of ethene and ethylene oxide. Carcinogenesis 10: 39-41.

32. Clemens MR, Remmer H (1982) Volatile alkanes produced by erythrocytes: an assay for in vitro studies on lipid peroxidation. Blut 45: 329-335.

33. Bolt HM (1996) Quantification of endogenous carcinogens. The ethylene oxide paradox. Biochem Pharmacol 52: 1-5

34. Cartoni A, Menna P, Salvatorelli E, Braghiroli D, Giampietro R, et al. (2004) Oxidative degradation of cardiotoxic anticancer anthracyclines to phthalic acids. Novel function or ferrylmyoglobin. J Biol Chem 279: 5088-5099.

35. Buczynski MW, Dumlao DS, Dennis EA (2009) Thematic Review Series: Proteomics. An integrated omics analysis of eicosanoid biology. J Lipid Res 50: $1015-1038$

36. Cormier SA, Taranova AG, Bedient C, Nguyen T, Protheroe C, et al. (2006) Pivotal Advance: eosinophil infiltration of solid tumors is an early and persistent inflammatory host response. J Leukoc Biol 79: 1131-1139.

37. Mattes J, Hulett M, Xie W, Hogan S, Rothenberg ME, et al. (2003) Immunotherapy of cytotoxic T cell-resistant tumors by T helper 2 cells: an eotaxin and STAT6-dependent process. J Exp Med 197: 387-393.

38. Schmid MC, Varner JA (2010) Myeloid cells in the tumor microenvironment modulation of tumor angiogenesis and tumor inflammation. J Oncol 2010 201026.

39. Ntayi C, Lorimier S, Berthier-Vergnes O, Hornebeck W, Bernard P (2001) Cumulative influence of matrix metalloproteinase-1 and -2 in the migration of melanoma cells within three-dimensional type I collagen lattices. Exp Cell Res 270: 110-118.

40. Hofmann UB, Westphal JR, Waas ET, Zendman AJ, Cornelissen IM, et al. (1999) Matrix metalloproteinases in human melanoma cell lines and xenografts: increased expression of activated matrix metalloproteinase-2 (MMP-2) correlates with melanoma progression. $\mathrm{Br} \mathrm{J}$ Cancer 81: 774-782. 